Document downloaded from:

http://hdl.handle.net/10251/100811

This paper must be cited as:

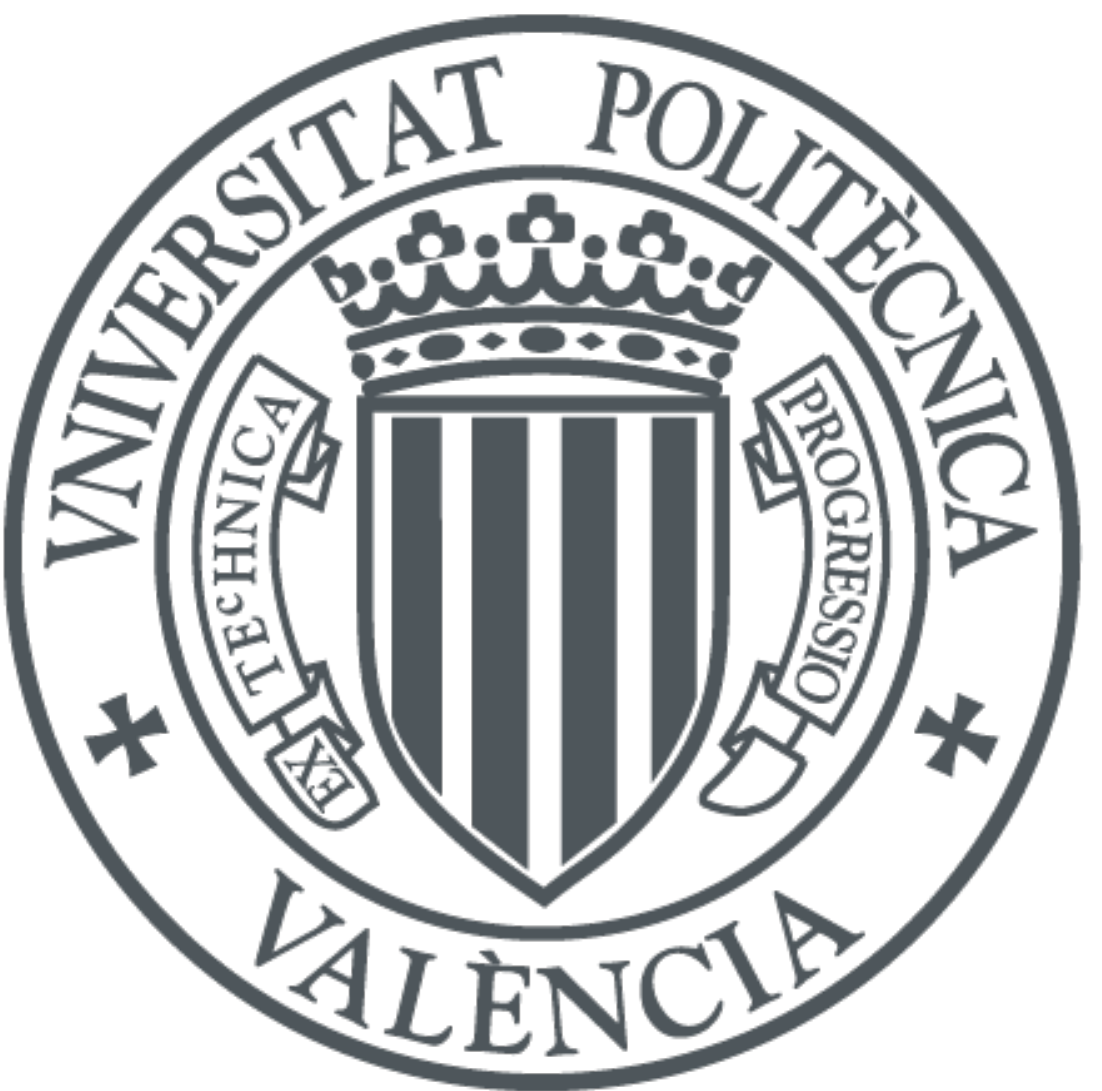

The final publication is available at

https://doi.org/10.1016/j.jcis.2011.05.013

Copyright Elsevier

Additional Information 


\title{
COATING TYPOLOGIES AND CONSTRAINED SWELLING OF HYALURONIC ACID GELS WITHIN SCAFFOLD PORES
}

M. Arnal-Pastor ${ }^{1}$, A. Vallés-Lluch ${ }^{1,2}$, M. Keicher ${ }^{1}$, M. Monleón Pradas ${ }^{1,2,3,{ }^{*}}$

${ }^{1}$ Center for Biomaterials and Tissue Engineering, Universitat Politècnica de València, Cno. de Vera s/n, 46022, Valencia, Spain

${ }^{2}$ Regenerative Medicine Unit, Centro de Investigación Príncipe Felipe, Av. Autopista del Saler 16, 46013 Valencia, Spain

${ }^{3}$ Networking Research Center on Bioengineering, Biomaterials and Nanomedicine, Valencia, Spain

*Corresponding author. Tel.: +34963877277; fax: +34963877276. E-mail: mmonleon@ter.upv.es

\begin{abstract}
A set of elastomeric scaffolds with a well defined porous structure was prepared with a template leaching procedure and coated with hyaluronic acid solutions. Depending on the coating process parameters the hyaluronic acid deposited on the pores had configurations ranging from thin disconnected aggregates to a thick continuous layer on the pore surface. The development of the coating layer was studied by scanning electron microscopy and the materials were subjected to dynamical and equilibrium swelling experiments in a water vapor ambient of fixed activity. The porosity change due to coating and to swelling of the coating layer were determined. The hyaluronic acid coating the pores has a different swelling capacity depending on the type of layer formed, as a consequence of the scaffold constraint and of the layer typology. These
\end{abstract}


factors were investigated analytically by modifying the standard theory of gel swelling. An experimental quantity is introduced which reflects the constrainment build-up on gel swelling.

Keywords: Scaffold; hyaluronic acid/hyaluronan; swelling

\section{Introduction}

Most cell-based and tissue engineering therapies need multifunctional scaffolds to locate stably a supply of cells and/or to provide specific cues such as spatial threedimensional organization, mechanical stimuli, local drug or factor delivery, etc. For many of the intended applications the sizes of these scaffolds become considerable (think of cardiac patches, large bone defects, etc). This fact further imposes the requirement that the scaffold be vascularizable, otherwise cells in the core of the construct will be too far from microvessels and lack oxygen and nutrient supplies, becoming non-viable. All these demands cannot be realistically met with singlecomponent scaffolds, and therefore combinations of several types of materials and structures must be designed for different specific purposes. One of the concepts of interest is to have a base scaffold providing a three-dimensional environment and mechanical properties whose inner pore surfaces are coated by a different material (such as a gel), which may have better cell-related properties and/or may lodge drugs or growth factors to be released in situ.

Such innerly coated scaffolds have been used, for example, by Blan et al. [1], who injected fibrin gel in the pores of a chitosan scaffold to enhance cardiomyocyte retention 
and observed active force in the combined construct upon electrical stimulation. Antunes et al. [2] coated with HA the pores of PLLA scaffolds. Filová et al. [3] developed a technique for coating scaffolds' surfaces with a fibrin film, based on the catalytic effect on the fibrinogen of previously surface-bound thrombin on the surfaces, preventing the clotting of the solution within the pores; they coated the pores of polylactide fibrous scaffolds to improve the development of endothelial cells [4]. Croll et al. [5] obtained multilayer coatings of hyaluronic acid (HA) and chitosan onto lactic acid/glycolic acid copolymers, which could be further functionalized covalently with collagen. Polini et al. [6] compared different collagen coatings on poly(methyl methacrylate) electrospun fibers obtained by physisorption and covalent binding, and found a dependence of cell attachment and proliferation on the type of linking. Bayraktar et al. [7] employed "aqueous coatings" of cross-linked silk fibroin obtained in several steps for drugs controlled release.

In this work we study the problem of coating the pores of a poly(ethyl acrylate), PEA, scaffold with hyaluronic acid, HA, in a controlled way, in order to obtain a potentially multifunctional construct. HA is a non-sulfated glycosaminoglycan prevalent in the extracellular matrix, ECM, of many tissues, where it plays an important role in the maintenance of extracellular space, the transport of ion solutes and nutrients and the preservation of tissue hydration, and mediates the inflammation and repair processes $[8,9]$. It is biodegradable, biocompatible, and non-immunogenic. HA has been previously employed as a tissue engineering scaffold [10, 11], drug carrier [12], and for other therapeutic applications [13]. Moreover, the low molecular weight degradation products of HA stimulate angiogenesis and endothelial cell proliferation and migration [13-15]. As a scaffold we employ a PEA matrix with cylindrical pores in three 
orthogonal space directions [16]. Its spatial regularity and the shape of the pores may be of help in promoting the formation and organizing the growth of new blood vessels. Its layered structure, moreover, may be of interest in applications which require resistance to normal stresses. The polymer PEA is a hydrophobic elastomer at body temperature, and thus is able to recover after applied stresses; it has an optimal biological performance, as assessed in previous works with different scaffold structures and cell types such as chondrocytes [17], fibroblasts [18], endothelial cells [19], dental pulp stem cells [20], conjunctival cells [21] and neural cells [22, 23].

Depending on the amount of gel adsorbed and on the arrangement of the gel layer, different typologies of the scaffold coating can be imagined, see Figure 1. All of them seem worth investigating, since they might be useful for different purposes. If the gel completely fills the scaffold's pores (Figure 1 a) it may act as an encapsulating medium for the cells, which are then kept isolated from each other and non-attached to the scaffold's surfaces, while the gel allows for nutrient diffusion and cellular waste removal; thus, a three-dimensional isolated cell arrangement is achieved, much in the manner chondrocytes are laid out in cartilage, for example. If the gel coats the pores' surfaces uniformly as a layer cells may adhere directly to the gel instead of doing it to the polymer's surface (Figure $1 \mathrm{~b}$, left); this may be a wanted situation in cases in which the cells prefer more hydrophilic and natural substrata, while the scaffold provides the mechanical consistency which the gel lacks. Additionally, the gel layer may be used as a medium for drug or factor release, with these molecules either directly trapped into the gel network or incorporated into microparticles, and these particles embedded in the gel (Figure $1 \mathrm{~b}$, right); as the gel layer swells or degrades the active molecules are released. Still another typology of interest is obtained when the gel is sparsely adsorbed as 
unconnected aggregates on the pores' surfaces (Figure $1 \mathrm{c}$ ). In this situation both the gel aggregates' and the scaffold surfaces are available for the cells to interact with. This opens a number of colonization and co-culture possibilities: different types of cells might have differential preferences for each one of the two media; adhered cells onto the scaffold's surface may be directly influenced by the release and degradation products of the gel aggregates, etc.

a)

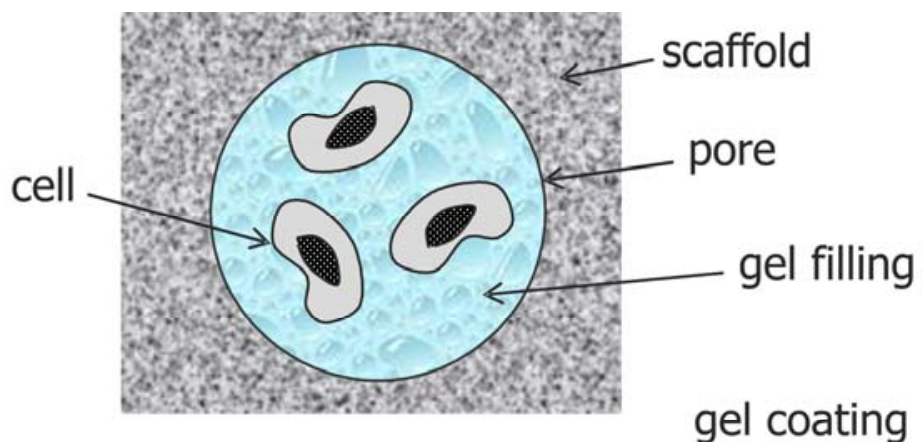

b)

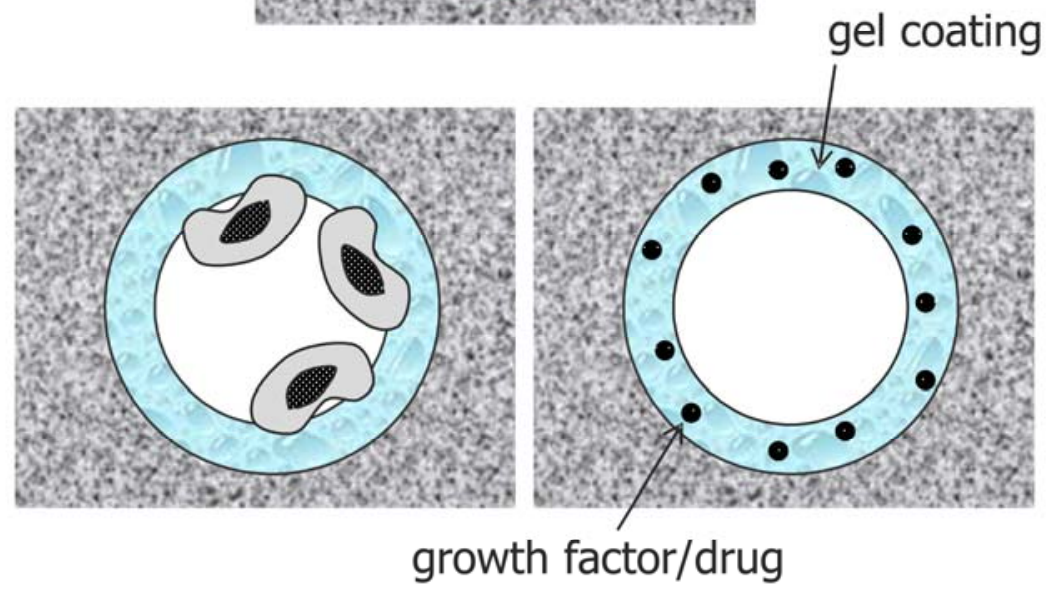

c)

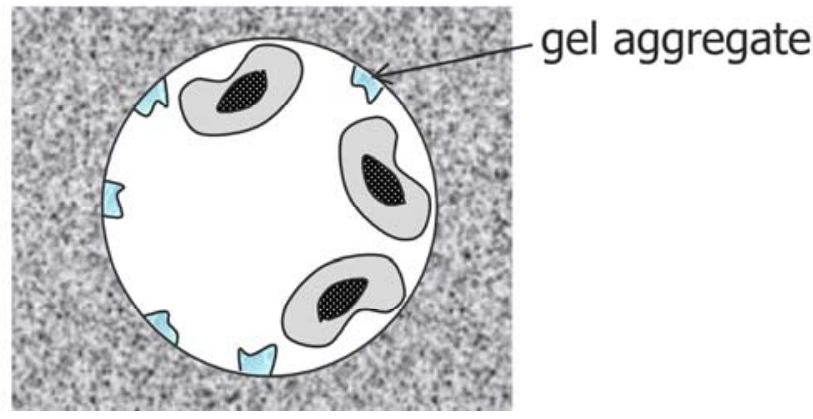

Figure 1. Sketch of different typologies of the configuration of a gel in the pores of a scaffold (see text). 
The purpose of the present work was to address the problems faced to produce the coating typologies just described, and to characterize their physicochemical state in the scaffold-coating construct. More specifically, answers were sought to questions such as: what is the adequate procedure to uniformly introduce an aqueous gel into a hydrophobic structure; what is the influence of solution concentration and of adsorption time on the type of coating obtained; how does the swelling capacity of the gel change as a consequence both of its typology (in the sense discussed above) and of its being constrained by the scaffold's elasticity; how does the system's porosity change with coating, how does porosity change when the gel swells inside the pores; and, finally, to what extent can the adsorbed HA be crosslinked in order to broaden its potential applications [12, 24-28], i.e., to prevent its rapid dissolution, to regulate its chemical stability, swelling, degradation and consequently its drugs release properties.

Though the system here investigated may be of concrete application in cardiac tissue engineering and in neural tissue engineering, the system is here regarded as a model to address the problems raised by the scaffold/coating complexes in general.

\section{Materials and methods}

\subsection{Materials}

Scaffolds of cylindrical orthogonal pores

The scaffolds here employed were fabricated following the method described in [16]. Ethyl acrylate (99\%, Aldrich), EA, monomer was mixed with 0.1 wt \% of azo-bis- 
isobutyronitrile (99\%, Fluka), AZBN, as thermal initiator, and 2 wt \% of ethylenglicoldimethacrylate (98\%, Aldrich), EGDMA, as crosslinker, stirred for $15 \mathrm{~min}$ and polymerized inside a porogen template produced by sintering 8 layers of nylon fabrics with nominal thread diameter equal to $150 \mu \mathrm{m}$ and mesh opening of $300 \mu \mathrm{m}$ (SAATI S.A., Barcelona, Spain) and placed between glass plates to obtain a 3-D mould. The previously described monomeric mixture was injected into these templates, polymerized for $24 \mathrm{~h}$ at $60^{\circ} \mathrm{C}$, and post-polymerized for $24 \mathrm{~h}$ more at $90^{\circ} \mathrm{C}$. Afterwards, the filled templates were cut into small pieces of approx. $20 \mathrm{~mm}$ x $20 \mathrm{~mm}$ x $1 \mathrm{~mm}$ and then rinsed in nitric acid (65\%, Scharlab) at ambient temperature under gentle shaking for $48 \mathrm{~h}$ in order to dissolve the fiber template. Finally, the obtained samples were washed in boiling ethanol for $40 \mathrm{~h}$, and dried $24 \mathrm{~h}$ at room temperature, a further $24 \mathrm{~h}$ under vacuum and room temperature, and $24 \mathrm{~h}$ more under vacuum at $60^{\circ} \mathrm{C}$. The scaffolds were stored in vacuo until use. A set of scaffolds was subsequently coated with hyaluronic acid, another set was employed bare for comparison.

\section{Hyaluronic acid solutions}

Hyaluronic acid (hyaluronic acid sodium salt, 99\%, Sigma, 1.63 MDa, obtained by fermentation of streptococcus equi bacteria), HA, was dissolved in 0,2 $\mathrm{M}$ sodium hydroxide (extrapure, Scharlau), $\mathrm{NaOH}$, aqueous solution, in order to obtain two solutions with different concentrations: $0.5 \mathrm{wt} \%$ and $5 \mathrm{wt} \%$. Eight drops of alcian blue (alcian blue 8Gx certified, Sigma) were added per $5 \mathrm{mg}$ of HA solution to stain the gels and make them visible within the scaffolds. After $24 \mathrm{~h}$ of stirring these solutions were employed to coat the pores of the scaffolds. 


\subsection{Methods}

\section{Hyaluronic acid coating}

Due both to the hydrophobicity of the elastomeric scaffolds and to their microporous structure, their filling with the aqueous HA solution had to be performed under vacuum in order to achieve an efficient penetration of the HA solutions into the pores. The scaffold samples were put in a $20 \mathrm{ml}$ syringe, the aqueous HA solution was loaded, and the air inside the syringe was removed. Then the luer taper of the syringe was sealed and the HA solution was forced to penetrate in the scaffolds by performing five repeated strokes of $4 \mathrm{ml}$ each time. Once soaked with the HA solutions the samples became blue and were then dried for $24 \mathrm{~h}$ at room conditions, by passing a thin thread transversally to the samples and hanging them, avoiding contact with any surface and exposing both faces to the ambient. This filling-drying process is referred to in what follows as "a cycle". Different thicknesses of the coating layer were achieved by performing from 1 up to 5 repeated cycles, both with the $0.5 \mathrm{wt} \%$ and the $5 \mathrm{wt} \% \mathrm{HA}$ solutions. The following nomenclature is hereafter employed to identify the samples subjected to the different coating procedures: 05HA\# and 5HA\# designate the scaffolds coated, respectively, with $0.5 \mathrm{wt} \%$ and $5 \mathrm{wt} \%$ HA solutions, \# being the number of cycles.

\section{Crosslinking of HA coatings}

A number of samples of the 5HA\# type was subjected to a process of crosslinking of the HA layer [26]. Firstly, the samples were immersed during $20 \mathrm{~min}$ in a 80:20 vol \% mixture of acetone (synthesis grade, Scharlau) and 0,005 $\mathrm{M} \mathrm{NaOH}$ (reagent grade, 
Scharlau) aqueous solution. Divinyl sulfone (97\%, Aldrich, 118.15 Da), DVS, was then added as crosslinker in a 7:3 DVS/HA molar ratio. $24 \mathrm{~h}$ later the scaffolds were gently washed with a $20 / 80$ vol $\%$ mixture of water/acetone for $30 \mathrm{~min}$, rinsed with distilled water and dried. To identify the crosslinked samples an $\mathrm{x}$ is added to the series label, thus: 5HA\#x ("sample coated with the 5wt \% HA solution a number \# of cycles and crosslinked with DVS").

\section{Morphological observation with SEM}

Both bare and vacuum-dried HA-coated scaffolds were examined with a JSM 6300 scanning electron microscope (JEOL Ltd., Tokyo, Japan), SEM, at $10 \mathrm{kV}$ of acceleration voltage. Samples were sputter-coated with gold. The HA-coated scaffolds were broken in liquid nitrogen in order to obtain cross section images.

\section{Determination of the HA adsorbed}

The amount of HA adsorbed into the pores of the PEA scaffolds was quantified by comparing the weights of dry samples bare and after the HA coating procedures. A Mettler AE 240 balance (Mettler-Toledo Inc., Columbus, OH, USA), with a sensitivity of $0.01 \mathrm{mg}$, was employed for this purpose. Measurements were performed at least 3 times for each type of sample. The amount of HA adsorbed into the scaffolds after the coating processes was quantified by the HA mass fraction, $\omega_{H A}$, defined as:

$$
\omega_{H A}=\frac{m_{H A}}{m_{H A}+m_{\text {bare }}}
$$

where $m_{\text {bare }}$ is the mass of the bare PEA scaffold and $m_{\mathrm{HA}}$ is the mass of (dry) HA adsorbed. 


\section{Determination of water sorption}

The water sorption capacity of the samples was evaluated in a vapor ambient at $37^{\circ} \mathrm{C}$ and $66 \%$ relative humidity (water activity equal to 0.66 ), $\mathrm{RH}$, as follows. Samples were placed in a tightly sealed test tube over a supersaturated potassium iodide (extrapure, Scharlau) aqueous solution [29], avoiding any contact of the samples with the solutions. The tubes were placed in an oven at $37^{\circ} \mathrm{C}$. After selected times the tubes were opened and the samples weighed until no weight change was further observed. The value obtained at the longest time, 5 days, was taken as the equilibrium value. The different quantities employed for characterizing the water sorption of the samples are defined as follows: for each time, the water content, $W C$, is the mass of water absorbed, $m_{\text {water, }}$ divided through the mass of dry sample consisting in the scaffold plus the coating, $m=$ $m_{\text {bare }}+m_{\mathrm{HA}}$, The equilibrium water content, $E W C$, is the value of $W C$ at 5 days. The swelling measurements were repeated with 3 samples for each type of scaffold.

\section{Porosity measurements}

The porosity of the dry 05HA\# and 5HA\# samples, and of the 5HA\#x samples equilibrated in $66 \%$ RH was determined gravimetrically with a Mettler AX 205 balance (Mettler-Toledo Inc., Columbus, OH, USA) equipped with a Mettler ME 33360 density accessory kit. The samples were weighed in air and immersed in $n$-octane (reagent grade

$98 \%$, Aldrich, $\rho_{\text {n-octane }}=0.703 \mathrm{gcm}^{-3}$ ) at room temperature. The porosity $\pi$ is defined as the pore volume fraction of the scaffolds,

$$
\pi=\frac{V^{\text {pore }}}{V^{\text {app }}}
$$


where $V^{p o r e}$ is the volume of pores and $V^{a p p}$ is the geometric, or apparent, volume of the sample. The porosity was obtained from weight measurements as follows:

$$
\pi=\frac{V^{\text {pore }}}{V^{a p p}}=1-\frac{V}{V^{a p p}}=1-\frac{m \cdot v}{V^{a p p}}
$$

where $V$ and $v$ are, respectively, the real total and specific volumes of the material. The apparent volume $V^{a p p}$ was calculated from the measured linear dimensions of the sample, and the real specific volume $v$ was determined according to Archimedes' principle as

$$
v=\frac{V_{\text {displaced }}}{m}=\frac{\left(m-m_{\text {in n-octane }}\right) / d_{n \text {-octane }}}{m}
$$

where $m_{\text {in n-octane }}$ is the weight of the sample immersed in n-octane, $d_{\text {n-octane }}$ is the density of $n$-octane provided by the supplier, and $m$ is the weight of the sample in air.

\section{Results}

\subsection{Morphological observation with SEM}

Figure 2 shows representative SEM images of the different systems produced, in their dry state. Figures $2 \mathrm{~A}$ and $2 \mathrm{~B}$ show the bare PEA scaffolds in a cross section and a frontal view, respectively. The frontal surface (Figure 2 B) shows the plane of the two families of parallel pores generated by the porogenic fabrics; perpendicular to them the third set of pores, the interconnections between layers generated by the sintering of the fabrics, can be also recognized as more or less circular throats. The view of the scaffold cross section (Figure 2 A) shows the different layers of cylindrical pores. 

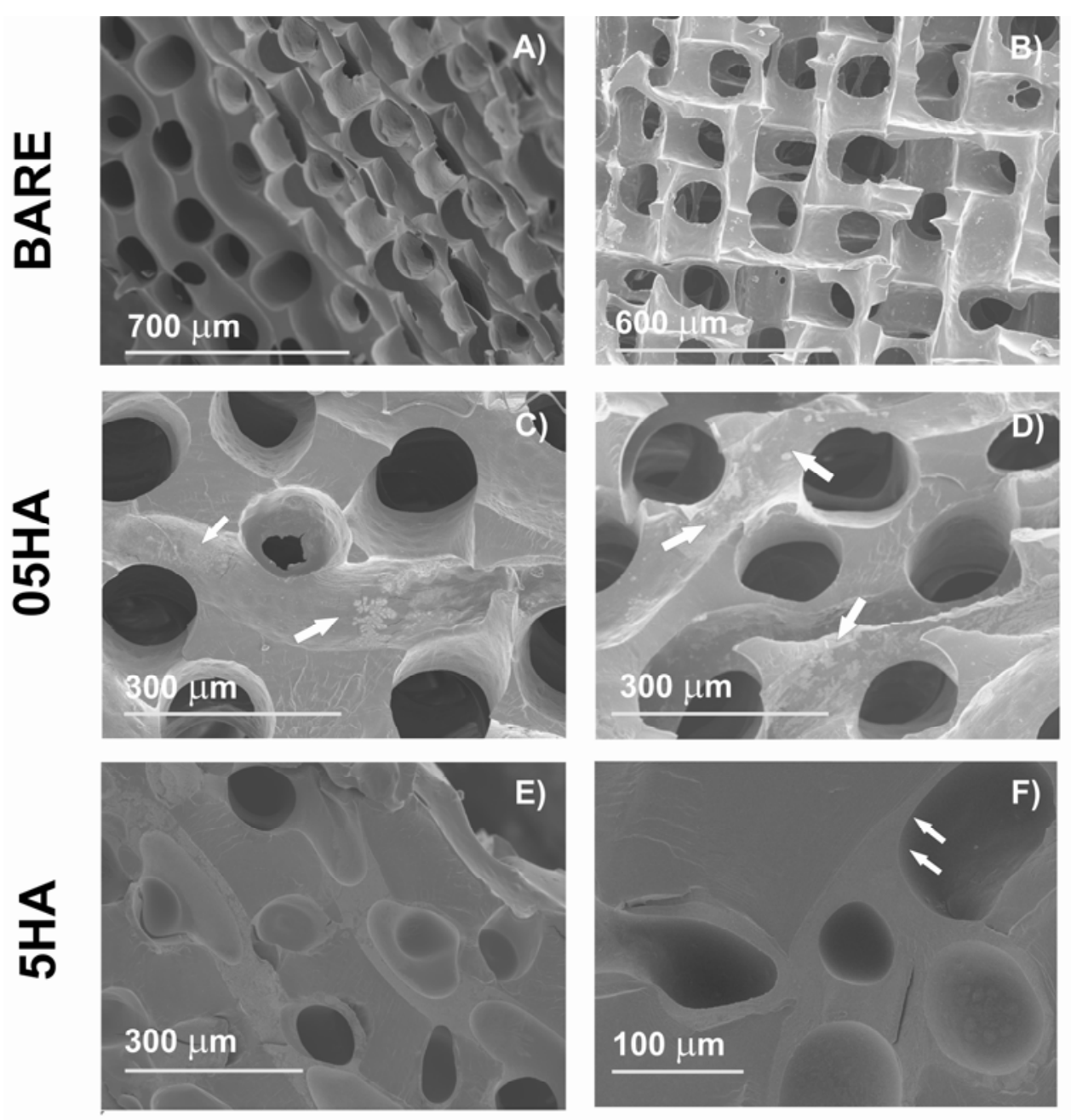

Figure 2. SEM images of the dry PEA scaffolds: (A,B) bare scaffold, cross section and frontal view, respectively; (C) 05HA1; (D) 05HA5; (E) 5HA2; (F) 5HA5. The arrows point at the adsorbed HA.

With one coating cycle the HA coating process with the $0.5 \mathrm{wt} \%$ HA solution produces aggregates of HA on the surface of the pores, as Figure $2 \mathrm{C}$ shows. These aggregates become more numerous as the number of cycles increases, but a uniform layer on the surfaces is not obtained even after 5 cycles with this solution concentration, Figure 2 D. By contrast, after the first cycle the coating with the $5 \mathrm{wt} \%$ HA solution already leads to a uniform continuous layer. The effect of further cycles is to increase the thickness of the layer, with the corresponding decrease in the pore diameter as well as the clogging 
of some pores, as observed in Figure 2 E. After the fifth cycle, Figure 2 F, the channels are filled with HA to a high degree.

\subsection{Amount of adsorbed HA as a function of concentration and number of cycles}

Figure 3 shows how the amount of adsorbed HA increases with the number of filling cycles both for the $0.5 \mathrm{wt} \%$ and the $5 \mathrm{wt} \%$ HA solutions. The PEA scaffold adsorbed around $7 \%$ of HA in the first cycle with $0.5 \mathrm{wt} \%$ HA solution. Afterwards, the amount of HA absorbed per cycle increases linearly with a gradient of approximately $2 \%$ per cycle, except for the last cycle, when it seems to stabilize. A $5 \mathrm{wt} \%$ concentration of HA in the solution leads to greater values of adsorbed HA, as expected: the amount of HA adsorbed in the first cycle is now about $15 \%$ in both the non- and the crosslinked samples, followed by an increase of around $7 \%$ in the second cycle and then a linear increase of about $5 \%$ per cycle.

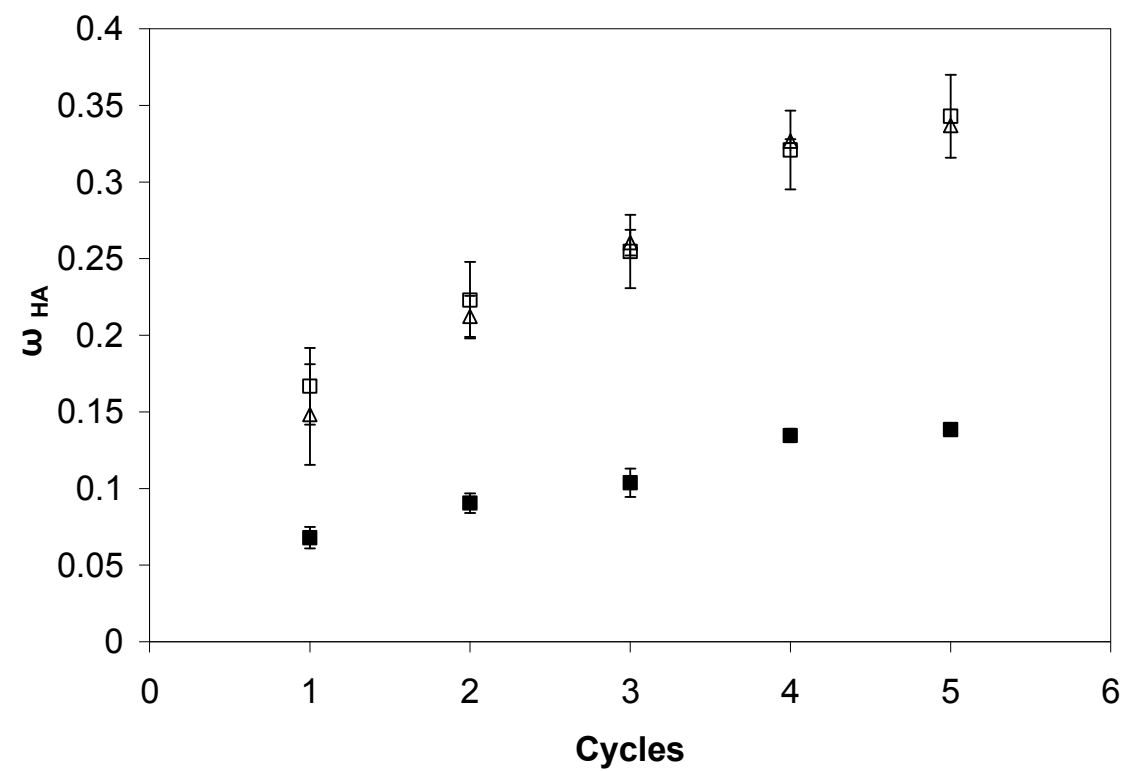

Figure 3. Mass fraction of $\mathrm{HA}, \omega_{H A}$, as a function of the number of cycles for: 05HA\#, $(\square)$ 5HA\#, and $(\triangle)$ 5HA\#x. 


\subsection{Water sorption at $66 \%$ relative humidity}

The inset in Figure 4 shows the time course of a representative sorption curve. The sample absorbed water rapidly in the first hours and then slowly reached equilibrium after about 5 days. Figure 4 contains the equilibrium values, $E W C$, of the coated samples. For the bare PEA scaffold, the $E W C_{\text {bare }}$ was $1.4 \%$. The samples with a crosslinked coating showed a significantly lower absorption (about $40 \%$ of that with non-crosslinked coating).

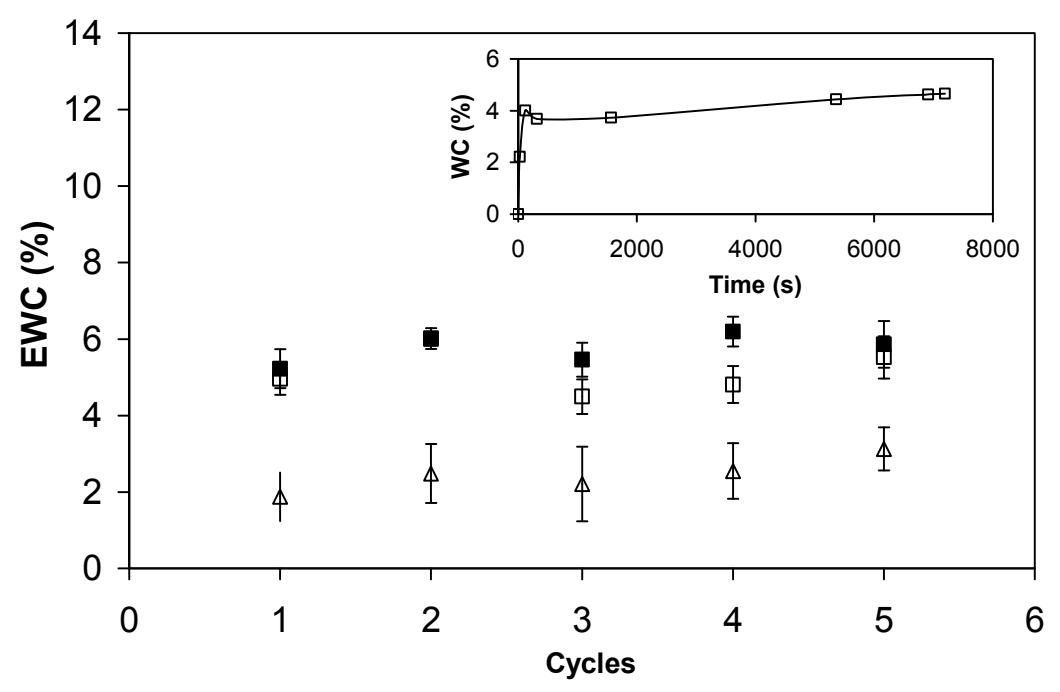

Figure 4. Equilibrium water content of the scaffold, $E W C$, as a function of the number

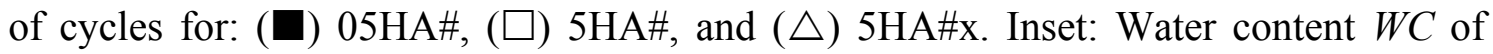
sample 5HA1 as a function of time.

\subsection{Porosity results}

Figure 5 shows the pore volume fraction of the samples as a function of the amount of adsorbed HA. The porosity of the bare scaffolds was around $75 \%$ when dry. The figure 
shows the results in the dry state for the non-crosslinked 05HA\# and 5HA\# series and the results for the swollen crosslinked series 5HA\#x. As expected, porosity decreases with increasing amount of coating, up to a $40 \%$ in the case of sample $5 \mathrm{HA} 5$, or a $25 \%$ in sample 5HA5x. The decrease is monotonous and almost linear in the case of the dry series; in the swollen series the drop stabilizes after a certain mass fraction of HA around $\omega_{H A}=0.20$. The porosity of the swollen samples was always markedly smaller than that of the dry samples.

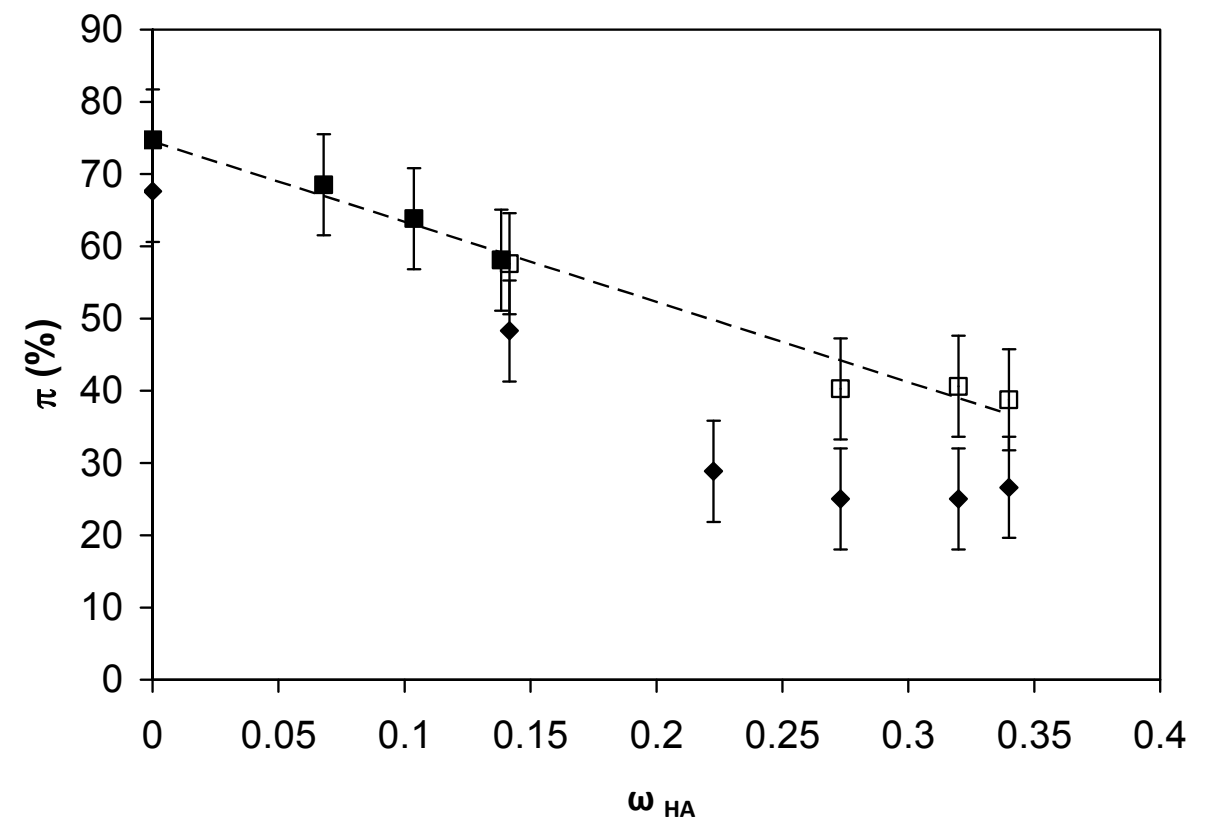

Figure 5. Porosity, $\pi$, as a function of the mass fraction of HA, $\omega_{H A}$, for: ( $\left.\mathbf{0}\right) 05 \mathrm{HA}$, $(\square)$ 5HA\#, and $(\bullet)$ 5HA\#x swollen in a $66 \%$ relative humidity atmosphere.

\section{Discussion}

These experimental results help to shed some light on the questions raised at the end of the Introduction. We address them here explicitly. 


\subsection{Pore coating procedure}

The PEA scaffolds studied in this work have a porous structure constituted by mutually orthogonal cylindrical pores resulting from the empty spaces left by the porogen template [16]. This structure can be recognized in Figure $2 \mathrm{~A}, \mathrm{~B}$, where the typical dimensions of the pores can be clearly identified. As a polymer, PEA is a relatively hydrophobic material; this fact, together with the hydrophilic and highly viscous nature of the HA solutions and the capillarity effects due to the pore diameters represent a set of obstacles in the way of getting a uniform filling of the porous structure. In the present work these difficulties were overcome by employing solutions of the HA polymer in aqueous $\mathrm{NaOH}$ in order to achieve lower viscosities [30], and by helping the filling with vacuum, as described in the Materials \& Methods section. Both the results of weighing and of SEM observations confirm that the scaffolds could be satisfactorily coated with HA using the described method. In order to have a control on the continuous growth of the thickness of the coating layer two parameters were varied: the concentration of the HA solution, and the number of consecutive filling-drying cycles to which the sample was subjected. By modifying both parameters, it was easy to produce HA coatings with typologies ranging from scattered aggregates, to completely clogged channels, with a uniform coating for the intermediate situations, Figure 6. 

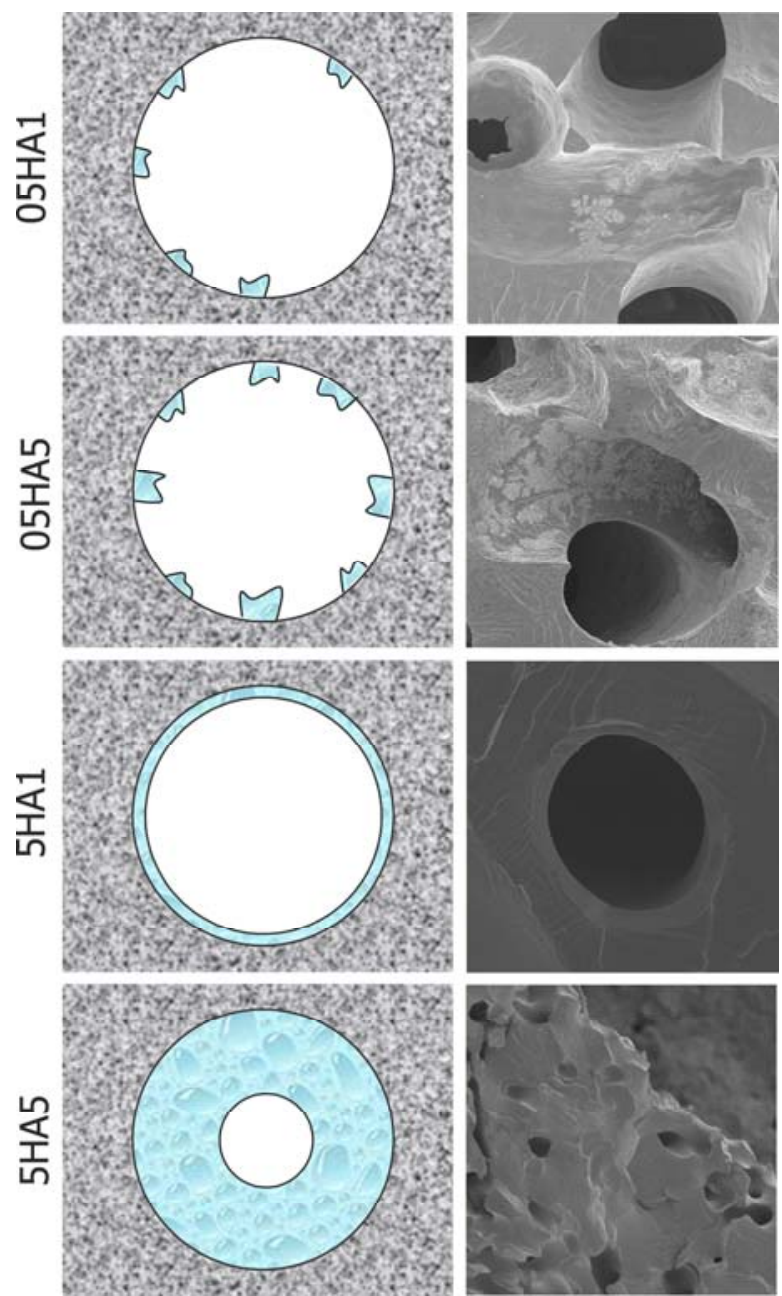

Figure 6. Scheme of the development of the HA coating in the scaffolds with the concentration in the HA solution and the number of cycles, from sporadic aggregates to the complete filling of the pores.

4.2. Influence of the number of cycles and of the coating solution concentration on the typology of the adsorbed layer

The gain in weight of Figure 3 proves that HA is effectively adsorbed onto the pore surfaces of the scaffolds, obviously in a greater amount from the 5 wt \% HA solution than from the $0.5 \mathrm{wt} \%$ one, though much less than a tenfold increase. The dependence of the HA mass fraction on the number of cycles is almost linear in both cases. An increase of cycle number with the 5 wt \% solution leads to a steady increase in the 
layer's thickness, (Figure $2 \mathrm{E}$ and F). This different way of layer build-up as a function of HA solution concentration explains why samples 05HA5 and 5HA1 can have close HA weight fractions and porosities (see below, and Figure 5) though having a very dissimilar topology (a disconnected morphology in the first case, versus a continuous in the second). This will also explain the different specific swelling capacities of the coatings which obtain from each solution concentration (see below, and Figure 7). Since the last disconnected layer, for the 05HA5 sample, has a HA mass fraction of $\omega_{H A}=0.145$, and the sample 5HA1, already continuously coated, has $\omega_{H A}=0.167$, the transition between both topologies must lie somewhere around $\omega_{H A}=0.15$.

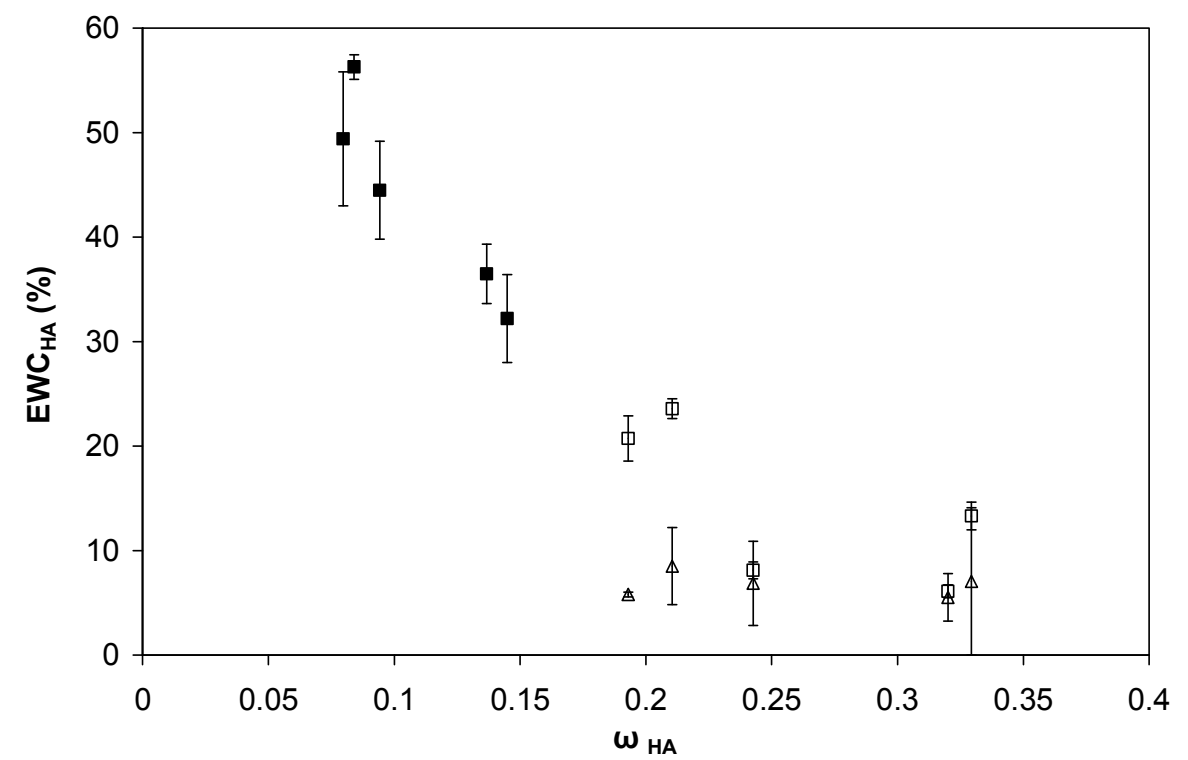

Figure 7. Equilibrium water content of the HA within the pores, $E W C_{H A}$, as a function

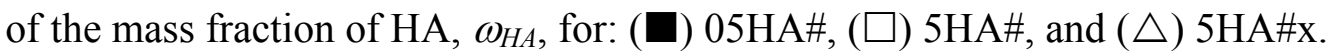

\subsection{Crosslinking of the gel inside the pores}

The HA coatings obtained from the adsorption of HA molecules from solution are soluble in water. Sorption experiments on the coated scaffolds performed in a saturated water vapor atmosphere resulted in the HA gel leaking from the pores due to its 
enormous degree of swelling; in a liquid environment this would have meant the rapid and complete dissolution of the coating. Though this may be a desired situation in some cases, other applications certainly require a more stable coating, not only to preserve it for longer times, but also to avoid an exaggerated swelling of the gel which could clog up the lumen of the pores. It was therefore decided to crosslink the HA within the pores once the layer had been adsorbed, by the two-step process with DVS described in the Materials \& Methods section. This process involves rehydrating the HA layer with the DVS solution, and thus could imply some loss of HA mass due to dissolution. However, because of the very fast crosslinking reaction with DVS [26] no significant mass difference could be detected before and after crosslinking and rinsing, which may give a first indication of the efficiency of the crosslinking process; another, more decisive one, is given by the equilibrium swelling data, which record the marked decrease of the EWC of the crosslinked samples when compared to the non-crosslinked (Figure 4).

\subsection{Changes in porosity due to coating of the pores' surfaces}

The expected change of the porosity $\pi$ of the coated dry scaffolds with an increasing mass fraction of HA in the scaffold can be deduced to be

$$
\begin{aligned}
\pi & =\frac{V^{\text {pore }}}{V^{\text {app }}}=\frac{V^{a p p}-V_{\text {bare }}-V_{H A}}{V^{a p p}}=\pi_{\text {bare }}-\frac{m \cdot \omega_{H A} / d_{H A}}{V^{a p p}}=\pi_{\text {bare }}-\frac{m_{\text {bare }}}{\left(1-\omega_{H A}\right) \cdot V^{a p p} \cdot d_{H A}} \cdot \omega_{H A}= \\
& =\pi_{\text {bare }}-\frac{d_{\text {bare }}^{\text {app }}}{d_{H A}} \cdot \frac{\omega_{H A}}{1-\omega_{H A}}
\end{aligned}
$$

where $m=m_{\text {bare }}+m_{H A}, \pi_{\text {bare }}$ is the porosity of the bare scaffold, ${ }_{\text {bare }}^{\text {app }}$ is the apparent density of the bare scaffold, and $d_{H A}$ is the density of dry HA. Since $y /(1-y)$ is a nearly linear function for values of $y$ less than 0.4 , according to the above equation the porosity of the system should fall linearly with $\omega_{H A}$, which is in fact what approximately 
happens, Figure 5; the experimental points for the coated scaffolds, moreover, extrapolate linearly to the experimental value of porosity of the bare scaffold, which is a good indication of the consistency of the results. In the case of a swollen coated scaffold the relationship between the porosity $\pi_{s w}$ and $\omega_{H A}$ is a bit more involved, but can still be established under mild simplifying assumptions and compared with the experiments. Let the subscript $s w$ distinguish the quantities associated with the swollen system, and let $\lambda_{s w}$, defined by $V_{s w}^{a p p}=\lambda_{s w}^{3} V^{a p p}$, be the-assumedly-isotropic stretch ratio of the swollen scaffold; then

$$
\begin{aligned}
\pi_{s w} & =\frac{V_{s w}^{\text {pore }}}{V_{s w}^{a p p}}=\frac{V_{s w}^{a p p}-V_{\text {bare }}-V_{H A}-V_{H_{2} O}}{V_{s w}^{a p p}}= \\
& =\lambda_{s w}^{-3}\left(\frac{\lambda_{s w}^{3} V^{a p p}-V_{\text {bare }}}{V^{a p p}}-\frac{m}{V^{a p p} d_{H A}} \cdot \omega_{H A}-\frac{m}{V^{a p p} d_{H_{2} O}} \cdot E W C\right)= \\
& =\lambda_{s w}^{-3}\left(\lambda_{s w}^{3}-1+\pi_{\text {bare }}-\frac{d_{\text {bare }}^{\text {app }}}{d_{H A}} \cdot \frac{\omega_{H A}}{1-\omega_{H A}}-\frac{d_{\text {bare }}^{\text {app }}}{d_{H_{2} O}} \cdot \frac{E W C}{1-\omega_{H A}}\right) .
\end{aligned}
$$

For stretching ratios of the scaffold close to unity (here the case) one may assume

$$
\begin{aligned}
& \lambda_{s w} \approx \lambda_{s w}^{-3} \approx \lambda_{s w}^{3} \approx 1, \text { and the above expression simplifies to } \\
& \pi_{s w}=\pi_{\text {bare }}-\frac{d_{\text {bare }}^{\text {app }}}{d_{H A}} \cdot \frac{\omega_{H A}}{1-\omega_{H A}}-\frac{d_{\text {bare }}^{\text {app }}}{d_{\mathrm{H}_{2} \mathrm{O}}} \cdot \frac{E W C}{1-\omega_{H A}}=\pi-\frac{d_{\text {bare }}^{\text {app }}}{d_{\mathrm{H}_{2} \mathrm{O}}} \cdot \frac{E W C}{1-\omega_{H A}} ;
\end{aligned}
$$

if, further, the water gain is referred to the mass of HA in the system by defining

$$
E W C_{H A} \equiv \frac{m_{\text {water in } H A}}{m_{H A}}=\frac{m \cdot E W C-m_{\text {bare }} \cdot E W C_{\text {bare }}}{m_{H A}}=\frac{E W C-\left(1-\omega_{H A}\right) \cdot E W C_{\text {bare }}}{\omega_{H A}},
$$

then we have

$$
\pi_{s w}=\pi-\frac{d_{\text {bare }}^{a p p}}{d_{\mathrm{H}_{2} \mathrm{O}}} \cdot\left(\frac{\omega_{H A}}{1-\omega_{H A}} \cdot E W C_{H A}+E W C_{\text {bare }}\right)=
$$




$$
=\pi-\frac{d_{\text {bare }}^{\text {app }}}{d_{\mathrm{H}_{2} \mathrm{O}}} \cdot E W C_{\text {bare }}-\frac{d_{\text {bare }}^{\text {app }}}{d_{\mathrm{H}_{2} \mathrm{O}}} \cdot E W C_{H A} \cdot \frac{\omega_{H A}}{1-\omega_{\mathrm{HA}}} .
$$

This equation tells, on the one hand, that the porosity of the swollen system is less than that of the dry system (for the same amount of HA in the scaffold), and, on the other hand, that the fall of porosity with increasing HA content in the scaffold is no longer proportional to $\omega_{H A}$, unless $E W C_{H A}$ itself varies linearly with $\omega_{H A}$ (which, as we shall see later, is not the case). Both predictions are confirmed by the experimental data of the swollen 5HA\#x series in Figure 5: the porosities of the swollen systems lie always below those of the dry systems of similar HA content, and the linearity of the drop is lost. An explanation for this non-linearity must take into consideration the changes in the specific hydrophilicity $E W C_{H A}$ of HA due to its typology.

4.5. The effect of confinement upon the water sorption capacity of the gel inside the pores of the scaffold

The different types of layers obtained by changing the HA solution concentration and the number of filling cycles have different specific sorption capacities. To see this, the water sorption data of Figure 4 were reworked to refer the amount of water sorbed solely by HA to the mass of HA in the sample: the parameter $E W C_{H A}$ defined in the previous paragraph gives the specific (per unit HA mass) water sorption capacity of HA in the different series of samples. Figure 7 shows the dependence of $E W C_{H A}$ on $\omega_{H A}$ for the series 05HA\#, 5HA\# and 5HA\#x. Remarkably, $E W C_{H A}$ is not a constant, as it would have been had the unit mass of HA behaved in all the samples with independence of the topology of the layer's configuration. A first steep decrease of $E W C_{H A}$ up to a HA mass fraction of about 0.2 occurs, then smoothes and stabilizes for greater mass fractions. This means that, within the scaffolds, the more amount of HA coating the pores, the less 
water each HA unit mass is able to absorb. The crosslinked samples have specific hydrophilicities $E W C_{H A}$ below the non-crosslinked ones for the same amount of HA, as expected.

This behavior can be explained in terms of the interplay between the elasticity of PEA (scaffold constraint) and the increasing hindrance of gel swelling due to surface coverage build-up and the curvature of the confining space (confinement effect), see the situations sketched in Figure 8, by means of a thermodynamical analysis of swelling.

a)

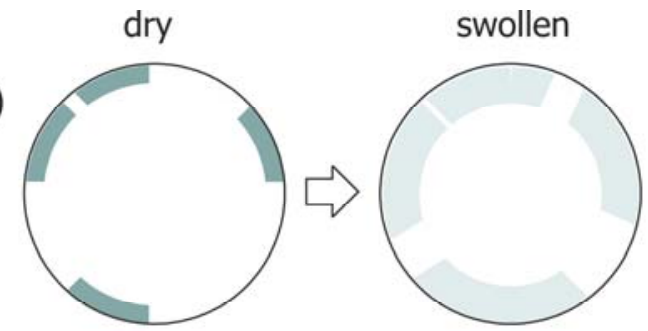

b)

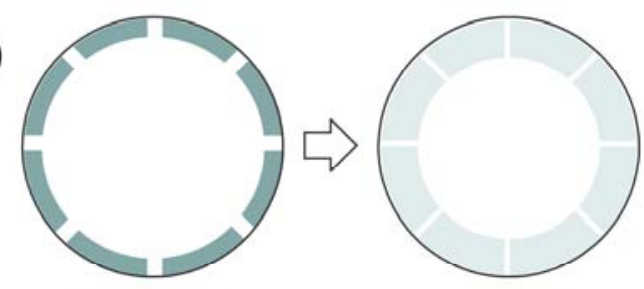

c)

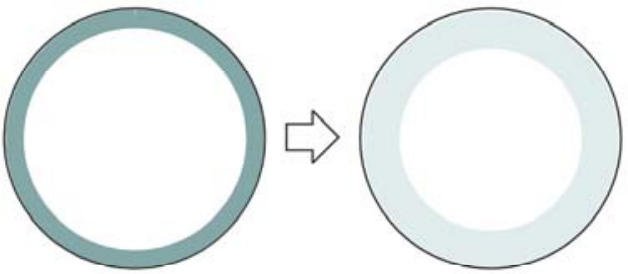

d)

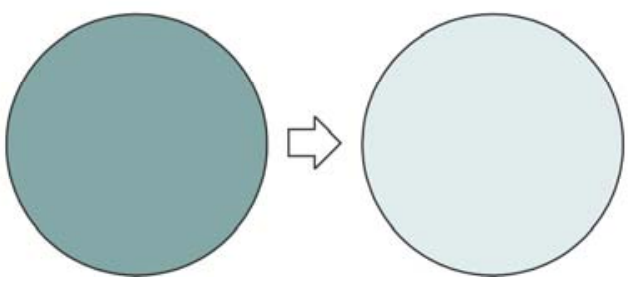

Figure 8. The confinement of the HA layer by the scaffold restricts in different ways the swelling capability of HA, depending on its typology.

Let the subscripts 1,2 and 3 identify in what follows the quantities associated with water, HA and the scaffold, respectively. If $\phi_{1}$ denotes the water volume fraction in the 
gel, the unconstrained gel in an environment of water activity $a_{1}^{e n v}=a^{*}$ reaches an equilibrium with a value $\phi_{1}=\phi_{1}^{*}$ determined by the equality of the chemical potential of water in the environment and within the gel: $\mu_{1}^{e n v}=\mu_{1}^{g e l}$, or, equivalently, $\mu_{1}^{e n v}-\mu_{1}=\mu_{1}^{\text {gel }}-\mu_{1} \equiv \Delta \mu_{1}^{\text {gel }}$. Here $\mu_{1}$ is the chemical potential of pure water, $\mu_{1}^{e n v}-\mu_{1}=R T \ln a_{1}^{e n v}$ and $R$ is the gas constant. If an equation of state for the excess chemical potential of water in the gel $\Delta \mu_{1}^{\text {gel }}=\Delta \mu_{1}^{\text {gel }}\left(T, p, \phi_{1}\right)$ is known (for example, the Flory-Rehner equation), then the equilibrium water volume fraction in the system $\phi_{1}^{*}$ is determined (the constants $T, p$ are hereafter omitted for simplicity):

$$
R T \ln a^{*}=\Delta \mu_{1}^{\text {gel }}\left(\phi_{1}^{*}\right) \quad \Rightarrow \quad \phi_{1}^{*}=\Phi_{1}^{\text {gel }}\left(a^{*}\right)
$$

where $\Phi_{1}^{\text {gel }}(a)$ denotes the inverse function of $\Delta \mu_{1}^{\text {gel }}\left(\phi_{1}\right)$.

In the case of constrained swelling in the same environment of water activity $a^{*}$ the system to be considered includes the gel (components 1: water, and 2: HA polymer) plus the scaffold (component 3 ). The equilibrium condition is now $\mu_{1}^{e n v}=\mu_{1}^{s y s}$, and $\mu_{1}^{\text {sys }}=\mu_{1}^{c . g e l}+\mu_{1}^{\text {scff }}$ with $\mu_{1}^{c . g e l}$ the chemical potential of water in the constrained gel and $\mu_{1}^{\text {scff }}$ the derivative of the elastic free energy of the scaffold with respect to the mol number of water in the system:

$$
\mu_{1}^{s c f f}=\frac{\partial G_{3}^{\text {elas }}}{\partial n_{1}} .
$$

Thus,

$$
R T \ln a^{*}=\mu_{1}^{e n v}-\mu_{1}=\mu_{1}^{s y s}-\mu_{1}=\Delta \mu_{1}^{c . g e l}+\mu_{1}^{s c f f}
$$

Again, this equation determines the equilibrium value $\phi_{1}^{* *}$ of water in the system once the equation of state of the constrained gel and that of the elastomer scaffold are known. According to the theory of rubber elasticity [31] an elastomer changes its Gibbs free 
energy when isotropically deformed as $\Delta G_{3}^{\text {elas }}=\frac{3}{2} n_{3} R T\left(\lambda_{3}{ }^{2}-1\right)$, where $n_{3}$ is the mol number of chains of the network material of the scaffold (PEA in our case) and $\lambda_{3}$ is the stretch ratio of the scaffold matrix elastomer. Since lodging more molecules of water within the gel implies stretching the scaffold, we have $\partial \lambda_{3} / \partial n_{1}>0$ and thus the function $f_{3}$ defined by

$\frac{\partial \Delta G_{3}^{\text {elas }}}{\partial n_{1}}=\frac{\partial \Delta G_{3}^{\text {elas }}}{\partial \lambda_{3}} \frac{\partial \lambda_{3}}{\partial n_{1}} \equiv R T \cdot \ln f_{3}\left(\phi_{1}\right)$

is always positive.

The Flory-Rehner theory of gel swelling admits for the unconstrained gel's free energy $G^{\text {gel }}$ contributions from the mixing of water and polymer (Flory-Huggins term) and from the elastic stretching of the polymer, $G^{\text {gel }}=G_{12}^{\text {mix }}+G_{2}^{\text {elas }}$, where the change in the elastic free energy of the gel is again given by the theory of rubber elasticity as $\Delta G_{2}^{\text {elas }}=\frac{3}{2} n_{2} R T\left(\lambda_{2}{ }^{2}-1\right)$, now with $n_{2}$ the mol number of gel chains and $\lambda_{2}$ the gel's stretch ratio due to its swelling, $\lambda_{2}=\left(1-\phi_{1}\right)^{-\frac{1}{3}}$. As revealed by the SEM pictures and discussed previously, the way in which HA adsorbs on the scaffold pore surfaces ranges from small disconnected clots to a uniform coating depending on the amount of HA adsorbed. The disconnected HA aggregates can swell almost freely not only in the direction normal to the pore's surface, but also laterally, and are constrained solely at their interface with the scaffold surface. As the number of HA aggregates on the pore surface increases the aggregates get closer, and their swelling starts implying lateral interactions. When the pore's surface is completely covered by the HA layer the free lateral expansion of the gel is completely constrained. Once this constraint has developed, the swelling of the layer is additionally influenced by the curvature of the 
pore surface, see the sketch in Figure 8, which sets a further limit to the sorption capacity of HA, since any increase in volume of the gel must result in an increase of the negative curvature of the gel's free (luminal) surface, paying a free energy cost. Thus, it must be assumed that the free energy of the constrained gel $G^{\text {c.gel }}$ must have, besides the mixing $G_{12}^{m i x}$ and the elastic $G_{2}^{\text {elas }}$ terms considered in the theory of unconstrained swelling, a further contribution $G^{\text {topol }}$ depending on a topological-and-form factor $\varphi$, such that

$G^{c . g e l}=G_{12}^{\text {mix }}+G_{2}^{\text {elas }}+G^{\text {topol }}=G^{\text {gel }}+G^{\text {topol }}$

and

$\frac{\partial \Delta G^{\text {topol }}}{\partial n_{1}}=R T \cdot \ln f\left(\phi_{1}, \varphi\right)>0$

holds. This topological-and-form factor $\varphi$ is a function of the HA content in the system and of other factors here left unspecified such as connectedness, degree of coverage, curvature of the interface, etc):

$\varphi=\varphi\left(\omega_{H A}, x\right), \quad x=\{$ connectedness, surface coverage, curvature, $\ldots\}$

it accounts for the influence on the gel's free energy of the topological features of the build-up of the scaffold-gel interface (connectedness, degree of coverage) and of the curvature increase of the swollen gel's free surface as a function of HA content in the scaffold. From (10) and (11) now

$$
\Delta \mu_{1}^{c . g e l}=\Delta \mu_{1}^{\text {gel }}+R T \cdot \ln f\left(\phi_{1}, \varphi\right),
$$

and thus, with (8),

$$
R T \cdot \ln a^{*}=\Delta \mu_{1}^{g e l}\left(\phi_{1}^{* *}\right)+R T \cdot \ln \left[f\left(\phi_{1}^{* *}, \varphi\right) \cdot f_{3}\left(\phi_{1}^{* *}\right)\right] .
$$


This is the wanted relationship between the constrained equilibrium swelling $\phi_{1}^{* *}$, the unconstrained gel equation of state $\Delta \mu_{1}^{\text {gel }}$, the elastomer equation of state $f_{3}$, and the constraint function $f\left(\phi_{1}, \varphi\right)$. Two of its consequences deserve consideration.

Firstly, (14) and (6) give

$\Delta \mu_{1}^{g e l}\left(\phi_{1}^{*}\right)=\Delta \mu_{1}^{g e l}\left(\phi_{1}^{* *}\right)+R T \cdot \ln \left[f\left(\phi_{1}^{* *}, \varphi\right) \cdot f_{3}\left(\phi_{1}^{* *}\right)\right] ;$

since thermodynamic stability [32] demands that $\Delta \mu_{1}^{\text {gel }}\left(\phi_{1}\right)$ be a monotonously increasing function of $\phi_{1}$, equation (15) implies that $\phi_{1}^{* *}<\phi_{1}^{*}$, or equivalently, $E W C_{H A}{ }^{* *}$ $<E W C_{H A}{ }^{*}$ for any fixed topology $\varphi$ of the gel coating. This proves that, as intuited, the constraint decreases the equilibrium swelling of the gel in a given environment with respect to the unconstrained swollen gel.

Secondly, the magnitude of the swelling decrement due to constrainment, $\Delta^{c} \phi_{1} \equiv \phi_{1}^{*}-\phi_{1}^{* *}$, can be proved to depend, for a fixed $a^{*}$, solely on the constraint functions $f_{3}$ and $\varphi$ : if equation (14) is solved for $\phi_{1}^{* *}$,

$\phi_{1}^{* *}=\Psi_{1}\left(a^{*}, \varphi, f_{3}\right)$,

then

$\Delta^{c} \phi_{1}=\Phi_{1}^{g e l}\left(a^{*}\right)-\Psi_{1}\left(a^{*}, \varphi, f_{3}\right)$

moreover, since, from (6) and (14),

$\Psi_{1}\left(a^{*}, \varphi, f_{3}\right)=\Phi_{1}^{g e l}\left(\frac{a^{*}}{f\left(\phi_{1}^{* *}, \varphi\right) \cdot f_{3}\left(\phi_{1}^{* *}\right)}\right)$,

the swelling decrement is completely determined by the constitutive properties of the unconstrained gel $\Phi_{1}^{\text {gel }}$ and the values of the constraints $f_{3}$ and $\varphi$.

$E W C_{H A}$ is a monotonously increasing function of $\phi_{1}$, and thus its dependences are the same as those given by (16): for a fixed environment activity $a^{*}$ 
$E W C_{H A}=F^{\text {constr }}\left(\varphi, f_{3}\right)$.

These results qualitatively account for the data represented on Figures 5 and 7. Equation (19) explains why, in the same environment of $a^{*}$, the specific hydrophilicities $E W C_{H A}$ of the different series of samples differ, Figure 7; it also explains the non-linear course of the swollen porosity data on Figure 5, since, from (5) and (19), porosity also depends on the constrainment:

$\pi_{s w}=\Pi^{\text {constr }}\left(\omega_{H A}, \varphi, f_{3}\right)$

Moreover, also because of equation (19), the plot of the quantity $E W C_{H A}$ against $\omega_{H A}$ (Figure 7) is, essentially, a plot of the constraint development as a function of HA increase in the coating. The total constraint on the HA gel develops steeply for approximately $\omega_{H A}<0.2$, and more smoothly for $\omega_{H A}>0.2$. This threshold coincides with the transition from an unconnected HA coating (series 05HA\#, filled squares in Figure 7) to a completely developed continuous HA layer (series 5HA\#, open squares in Figure 7). The difference between the $E W C_{H A}$ data of the $5 \mathrm{HA \#}$ series and the $5 \mathrm{HA \# \textrm {x }}$ series in Figure 7 is due, obviously, to the term $G_{2}^{\text {elas }}$ in (10), present in the 5HA\#x series but absent in the non-crosslinked 5HA\# series: crosslinking always decreases specific swelling relative to the non-crosslinked state.

In $(18,19)$ the function $f_{3}$ determines the part of the swelling decrement due to the scaffold elasticity; as read from its definition (9), it depends on the amount of scaffold stretch needed to accommodate new water molecules in the gel when swollen. Equations $(18,19)$ cannot discriminate the separate effects of $\varphi$ and $f_{3}$; nevertheless, a consideration of the situations depicted in the sketch of Figure 6 leads to the conjecture that scaffold elasticity will start playing a more important role after the complete development of a continuous HA layer, when the increased difficulty for lateral 
swelling of the gel forces the scaffold to stretch as an alternative competitive way to fit new water molecules, and be the predominant constraint in situations where the pores are completely filled. For a water activity of $a^{*}=0.66$, as was the case here studied, the amounts of water sorbed by the gel are not high, and thus $f_{3}$ cannot have a large effect; the nearly constant values of $E W C_{H A}$ for the crosslinked series in Figure 7 are a support of this consideration. On the whole, then, one may advance that, at this water activity, the course of $E W C_{H A}$ in Figure 7 is basically determined by the topological-and-form constraint on the swelling of HA.

\section{Conclusions}

Pore coatings by hyaluronic acid can be obtained in an ample typology variety by combining solution concentration and adsorption time, or number of filling-drying cycles. After the coating, the gel can be further effectively crosslinked to increase its stability and decrease its dimensional change upon swelling. Since each different typology may be of interest for a different application, a good characterization of the main physicochemical features of the different gel states produced seems worth undertaking. We found that the specific swelling capacity of hyaluronic acid and the porosity of the swollen coated scaffolds fall significantly when the amount of HA in the scaffold increases. The drops in both quantities are related to each other, and reflect the increasingly constrained swelling of the HA gel due to its confinement by an elastic, curved and limited space, the scaffold. The peculiar effects of these factors, mechanical and topological in nature, can be understood by analyzing the thermodynamics of swelling of the tripartite system water + gel + scaffold, modified to account for connectivity effects at the gel/scaffold interface and curvature effects at the free gel interface. It is shown in what way the decrease of the specific water uptake capacity of 
the HA gel in the scaffold with respect to that of the free HA gel is uniquely determined by the combination of both constraining effects.

\section{Acknowledgements}

The authors acknowledge the support of the FP7 NMP3-SL-2009-229239 project "Regeneration of cardiac tissue assisted by bioactive implants (RECATABI)". MMP further acknowledges the support of the Spanish Science \& Innovation Ministry through project MAT2008-06434. Roberto García Gómez is thanked for his help in preparing the bare scaffolds.

\section{References}

1. N.R. Blan, R.K. Birla, J. Biomed. Mater. Res. 86A (2008) 195-208.

2. J.C. Antunes, J.M. Oliveira, R.L. Reis, J.M. Soria, J.L. Gómez-Ribelles, J.F. Mano, J. Biomed. Mater. Res. Part A 94A (2010) 856-869.

3. T. Riedel, E. Brynda, J.E. Dyr, M. Houska, J. Biomed. Mater. Res. A 88 (2009) 437447.

4. E. Filová, E. Brynda, T. Riedel, L. Bacáková, J. Chlupác, V. Lisá, et al, J. Biomed. Mater. Res. A 90 (2009) 55-69.

5. T.I. Croll, A.J. O’Connor, G.W. Stevens, J.J. Cooper-White, Biomacromolecules 7 (2006) 1610-1622.

6. A. Polini, S. Pagliara, R. Stabile, G.S. Netti, L. Roca, C. Prattichizzo, et al, Soft Matter 6 (2010) 1668-1674. 
7. O. Bayraktar, Ö. Malay, Y. Özgarip, A. Batigün, Eur. J. Pharm. Biopharm. 60 (2005) 373-381.

8. J.R.E. Fraser, T.C. Laurent, U.B.G. Laurent, J. Intern. Med. 242 (1997) 27-33.

9. Y. Luo, K.R. Kirker, G.D. Prestwich, in: A. Atala, R.P. Lanza (Eds.). Methods of tissue engineering, San Diego, Elsevier, 2002, pp. 539-540.

10. S.A. Zawko, S. Suri, Q. Truong, C.E. Schmidt, Acta Biomat. 5 (2009) 14-22.

11. T.W. Wang, M. Spector, Acta Biomat. 5 (2009) 2371-2384.

12. Y. Luo, K.R. Kirker, G.D. Prestwich, J. Controlled Release 69 (2000) 169-184.

13. J. Gaffney, S. Matou-Nasri, M. Grau-Olivares, M. Slevin, Mol. Biosyst. 6 (2010) 437-443.

14. A. Sattar, P. Rooney, S. Kumar, D. Pye, D.C. West, I. Scott, P. Ledger, J. Invest. Dermatol. 103 (1994) 576-579.

15. X. Shen, K. Tanaka, A. Takamori, Artif. Organs 33 (2009) 781-787.

16. J.C. Rodríguez Hernández, A. Serrano Aroca, J.L. Gómez Ribelles, M. Monleón Pradas, J. Biomed. Mater. Res. B Appl. Biomater. 84B (2008) 541-549.

17. M. Pérez Olmedilla, N. García-Giralt, M. Monleón Pradas, P. Benito Ruiz, J.L. Gómez Ribelles, E. Cáceres Palou, et al, Biomaterials 27 (2006) 1003-1012.

18. D. Gugutkov, G. Altankov, J.C. Rodríguez Hernández, M. Monleón Pradas, M. Salmerón Sánchez, J. Biomed. Mater. Res. A 92 (2010) 322-331.

19. A.J. Campillo-Fernández, R.E. Unger, K. Peters, S. Halstenberg, M. Santos, M. Salmerón Sánchez, et al, Tissue Engineering A 15 (2009) 1331-1341.

20. A. Vallés, E. Novella, M. Sancho, G. Gallego, M. Monleón, C. Carda, in: R.M. Natal Jorge, S.M. Santos, J.M.R.S. Tavares, M.A.P. Vaz, J.C. Reis Campos (Eds.), Biodental Engineering, London, CRC Press, 2010, pp. 213-217. 
21. A.J. Campillo-Fernández, S. Pastor, M. Abad-Collado, L. Bataille, J.L. GómezRibelles, J.M. Meseguer-Dueñas, et al, Biomacromolecules 8 (2007) 2429-2436.

22. J.M. Soria, C. Martínez Ramos, O. Bahamonde, D. García Cruz, M. Salmerón Sánchez, M.A. García Esparza, et al, J. Biomed. Mater. Res. 83A (2007) 463-470.

23. C. Martínez-Ramos, S. Lainez, F. Sancho, M.A. García Esparza, R. Planells-Cases, J.M. García Verdugo, et al, Tissue Engineering A 14 (2008) 1365-1375.

24. A. Sannino, S. Pappadà, M. Madaghiele, A. Maffezzoli, L. Ambrosio, L. Nicolais, Polymer 46 (2005) 11206-112012.

25. T. Segura, B.C. Anderson, P.H. Chung, R.E. Webber, K.R. Shull, L.D. Shea, Biomaterials 26 (2005) 359-371.

26. M.N. Collins, C. Birkinshaw, J. Appl. Polym. Sci. 104 (2007) 3183-3191.

27. M.N. Collins, C. Birkinshaw. J. Mater. Sci. Mater. Med. 19 (2008) 3335-3343.

28. M.N. Collins, C. Birkinshaw. J. Appl. Polym. Sci. 109 (2008) 923-931.

29. L. Greenspan. J. Res. Nat. Bur. Stand A Phys. Chem. 81A (1977) 89-96.

30. A. Maleki, A.L. Kjoniksen, B. Nyström. Macromol Symp 274 (2008) 131-140.

31. B. Erman, J.E. Mark, Structure and properties of rubberlike networks, New York, Oxford University Pres, 1997, pp. 54-57.

32. J.M. Prausnitz, R.N. Lichtenthaler, E. Gomes de Azevedo, Molecular Thermodynamics of Fluid-Phase Equilibria, New York, Prentice Hall, 1999, pp. 213293. 